\title{
Robust Adaptive Depth Control of Hybrid Underwater Glider in Vertical Plane
}

\author{
Ngoc-Duc Nguyen ${ }^{1}$, Hyeung-sik Choi ${ }^{2, *}$, Han-Sol Jin ${ }^{2}$, Jiafeng Huang ${ }^{2}$, Jae-Heon Lee ${ }^{2}$ \\ ${ }^{1}$ Department of Electrical and Information Engineering, Seoul National University of Science and Technology, Seoul, Korea \\ ${ }^{2}$ Department of Mechanical Engineering, Korea Maritime and Ocean University, Busan, Korea \\ Received 25 December 2019; received in revised form 22 March 2020; accepted 09 June 2020
}

DOI: https://doi.org/10.46604/aiti.2020.4142

\begin{abstract}
Hybrid underwater glider (HUG) is an advanced autonomous underwater vehicle with propellers capable of sustainable operations for many months. Under the underwater disturbances and parameter uncertainties, it is difficult that the HUG coordinates with the desired depth in a robust manner. In this study, a robust adaptive control algorithm for the HUG is proposed. In the descend and ascend periods, the pitch control is designed using backstepping technique and direct adaptive control. When the vehicle approaches the target depth, the surge speed control using adaptive control combined with the pitch control is used to keep the vehicle at the desired depth with a constant cruising speed in the presence of the disturbances. The stability of the proposed controller is verified by using the Lyapunov theorem. Finally, the computer simulation using the numerical method is conducted to show the effectiveness of the proposed controller for a hybrid underwater glider system.
\end{abstract}

Keywords: nonlinear robust adaptive control, depth control, hybrid underwater glider, buoyancy engine

\section{Introduction}

Underwater glider (UG) is the new type of autonomous underwater vehicle (AUV). UG has some advantages over the conventional AUV. The first innovation is that it can use low energy to survey the large area of ocean and another feature is that it can glide extremely quietly under the ocean. Therefore, there are many researches on this vehicle not only in oceanography but also in military purposes. In [1], a stable gliding condition was derived and an LQR controller was designed for a sawtooth depth of UG using the equilibrium point in the stable condition. A model predictive control was developed in [2] for UG to compensate for the drift due to external disturbances. In [2], the simulation and experiment studies were carried out to find the optimal pitch angle in gliding motion for the maximum speed. In [4], an energy optimal depth controller was developed for a long-range autonomous underwater vehicle with applications to hybrid UG during the level flight. A robust integral sliding mode with a super-twisting algorithm was proposed in [5] for the trajectory tracking problem of autonomous UG with environmental disturbances. In [6], the optimal control of an UG vehicle was proposed using Linear Quadratic Regulator strategy. A new optimal three-dimensional path planning method was developed in [7] for the minimum energy consumption for UGs. In [8], a self-searching optimal control was proposed for pitch-keeping control of UG during descent and ascent under ocean currents and noise disturbances. A novel control algorithm was presented in [9] using reinforcement learning with Active Disturbance Rejection Control (ADRC) and it was compared with the classical ADRC by simulation of OUC-III glider. In [10], a new hybrid heading tracking control algorithm was proposed using adaptive fuzzy incremental PID and anti-windup compensator to improve the heading control of UGs. A dynamic surface decoupling control algorithm using

* Corresponding author. E-mail address: hchoi@kmou.ac.kr

Tel.: +8210-5581-2971 
ADRC was proposed in [11] and sea trial results also were presented here with some improvements in overshoot and settling time. In [12], an LQR controller to maintain the sawtooth vertical movement in the gliding motion of UG was presented with the reduced order Luenberger observer for estimating the gravity center and buoyancy mass. In [13], to realize the precise navigation for the developed hybrid UG, an attitude reference system was composed of a ring laser gyroscope and a geomagnetic sensor was presented applying Extended Kalman Filter algorithm. In addition, a ray-type shape underwater glider with robust adaptive heading control using propellers was developed and tested in the sea trials [14]. Among those gliders, the depth control problem is still a challenging problem. The purpose of this study aims at the depth control of the HUG using a combination of the pitch and speed controls, robust to disturbances will be designed.

In this research, to contribute to the control design of the torpedo-shaped hybrid underwater glider (HUG) with buoyancy engine and propeller, the adaptive robust depth control is proposed for depth keeping control against unknown parameters and environmental disturbances. This controller is designed based on the Lyapunov stability theorem and the backstepping technique for underactuated dynamics like HUG. The hydrodynamic coefficients of this vehicle were obtained from [15] due to the similar torpedo shape and similar design dimension. The heave motion is controlled through pitch control and speed control with the line-of-sight guidance law. To see the effectiveness of this proposed controller for depth keeping control, the computer simulation using MATLAB is performed and the stable performance is guaranteed.

\section{Vertical Model of Hybrid Underwater Glider}

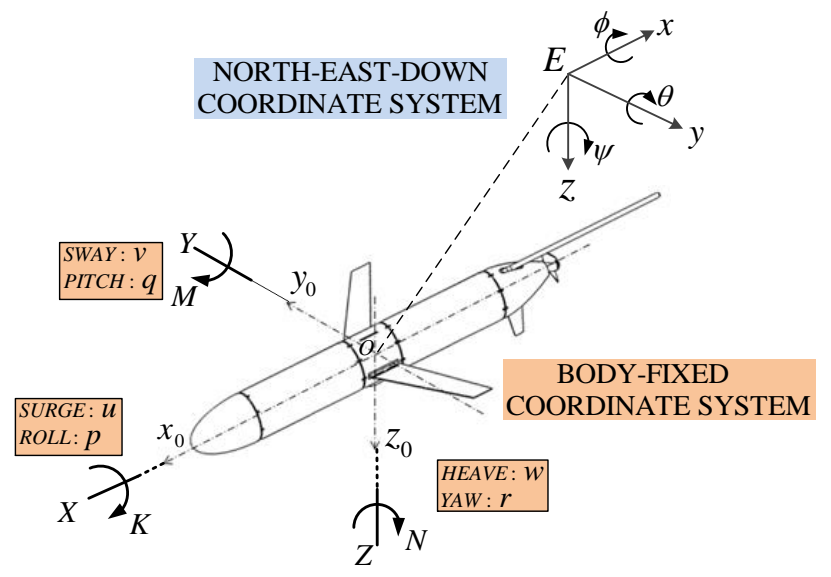

(a) earth-fixed and body-fixed coordinate system

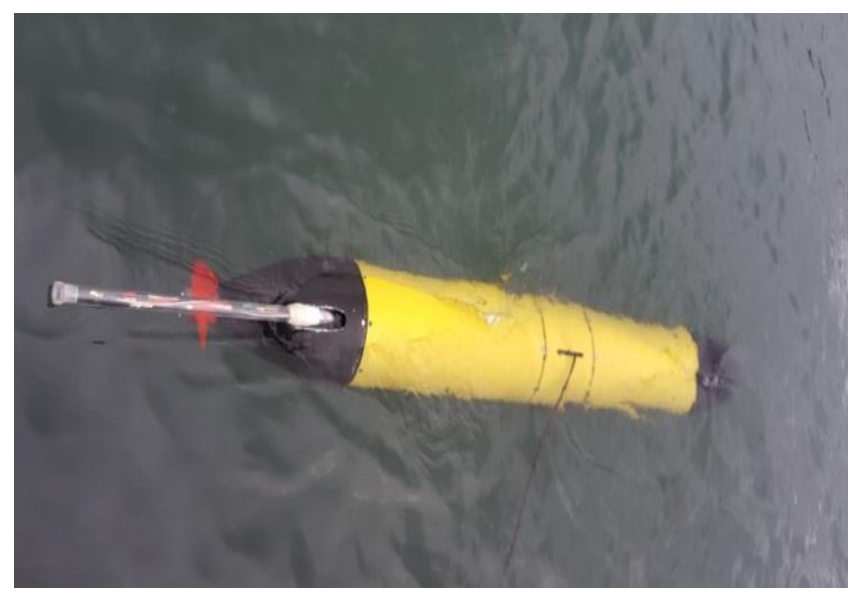

(b) developed HUG in the field test

Fig. 1 Hybrid underwater glider

To find the position of the UG; first, the relationship between the body-fixed coordinate and earth-fixed coordinate is expressed using the angle of the hull and the acceleration as in Fig.1. For this, heading and attitude sensors are in the glider [16]. The vertical dynamics of HUG can be expressed in the kinematic and kinetic model as Eq. (1) [17].

$$
\begin{gathered}
\dot{\eta}=J v \\
M \dot{v}+C(v) v+D(v) v+g(\eta)=\tau+J^{T} \tau_{e}
\end{gathered}
$$

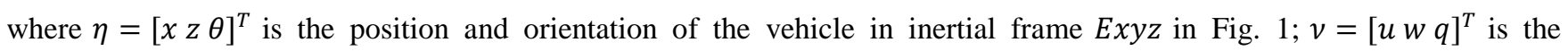
translation and angular velocity in the body-fixed frame $O X_{0} Y_{0} Z_{0}$ in Fig. 1 ; $J$ is Jacobian matrix; $M=M_{R B}+M_{A}$ is the inertia matrix; $C(v)=C_{R B}(v)+C_{A}(v)$ is the Coriolis and centripetal matrix; $D(v)$ is hydrodynamic damping matrix; $g(\eta)$ is the gravitational matrix; $\tau$ is the external forces and moments; $g_{0}(\eta)$ is the ballast forces and moments which are generated by buoyancy engine in the hybrid underwater glider (HUG); $\tau_{e}$ is the environment disturbances. To obtain the detail dynamic, one can expand Eq. (1) into Eq. (2), while the dynamic coefficients are obtained from the REMUS AUV model [15] which has the same hull dimension as the developed HUG. 


$$
\begin{aligned}
\dot{x} & =u \cos \theta+v \sin \theta \\
\dot{z} & =-u \sin \theta+v \cos \theta \\
\dot{\theta} & =q \\
\left(m-X_{\dot{u}}\right) \dot{u}= & -m z_{g} \dot{q}+m x_{g} q^{2}-m w q+Z_{\dot{w}} w q+Z_{\dot{q}} q^{2}+X_{|u| u}|u| u-(W-B) \sin \theta+\tau_{w} \sin \theta+\tau_{u}+\tau_{e u} \\
\left(m-Z_{\dot{w}}\right) \dot{w}= & m x_{g} \dot{q}+Z_{\dot{q}} \dot{q}+m z_{g} q^{2}+m u q-X_{\dot{u}} u q+Z_{|w| w}|w| w+(W-B) \cos \theta+\tau_{w} \cos \theta+\tau_{e w} \\
\left(I_{y y}-M_{\dot{q}}\right) \dot{q}= & -m z_{g}(\dot{u}+q w)+m x_{g}(\dot{w}-q u)+M_{\dot{w}} \dot{w}-Z_{\dot{w}} w u-Z_{\dot{q}} q u+X_{\dot{u}} u w+M_{|q| q}|q| q \\
& -\left(z_{g} W-z_{b} B\right) \sin \theta-\left(x_{g} W-x_{b} B\right) \cos \theta+\tau_{q}+\tau_{e q}
\end{aligned}
$$

As this time, $X_{\dot{u}}, Z_{\dot{w}}$, and $M_{\dot{q}}$ are the added mass coefficients; $X_{u}, Z_{w}$, and $M_{q}$ are linear damping coefficients; $X_{|u| u}$, $Z_{|w| w}$, and $M_{|q| q}$ are nonlinear damping coefficients; $W$ and $B$ are the weight and buoyancy force respectively in the neutral buoyancy condition; $x_{g}$ and $z_{g}$ are coordinates of gravity center in the body-fixed frame; $x_{b}$ and $z_{b}$ are coordinates of buoyancy center in the body-fixed frame; $\tau_{w}$ is the control force from buoyancy engine; $m$ and $I_{y y}$ are the vehicle mass and the y-axis inertia; $\tau_{q}$ is the control moment from mass-shifter; $\tau_{w}$ is the buoyancy force generated by the pump inside HUG body; $\tau_{u}$ is the thruster force; $\tau_{e u}, \tau_{e w}$, and $\tau_{e q}$ are disturbances from ocean currents and wave and $\left[\tau_{e u} \tau_{e w} \tau_{e q}\right]^{T}=J^{T} \tau_{e}$.

Sealed at atmosphere pressure, 1atm

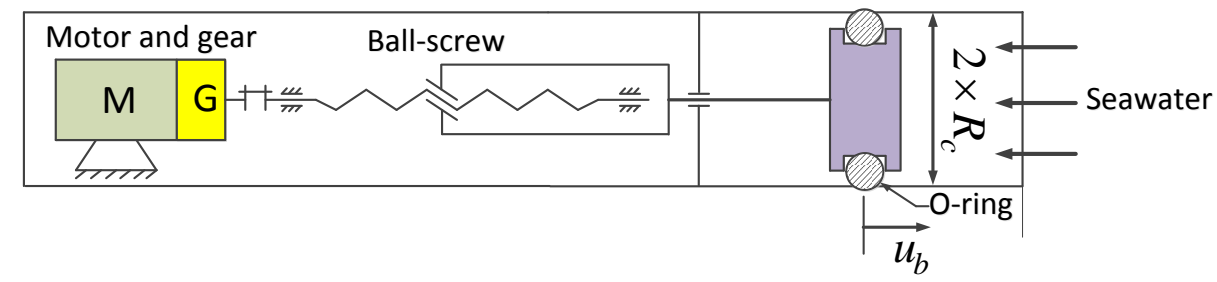

Fig. 2 Buoyancy engine diagram

In this HUG system, the buoyancy engine will let the water in or out by moving piston along the cylinder as revealed in Fig. 2. During this process, the volume of this vehicle will decrease or increase according to the position of the piston. If the weight is equal to the buoyancy force in the neutral condition, this glider descends when its volume is reduced and ascends toward the water surface when its volume is increased. In order to specify the force that this buoyancy engine can produce, the travel distance of the piston and the radius of cylinder should be defined. The buoyancy force is equal to the weight of seawater going in or out and it is indicated in Eq. (3). As this point, in Fig. 2, $u_{b}$ is the position of piston; $R_{c}$ is the radius of the cylinder; $\rho$ is the density of seawater; $g$ is the gravitational acceleration. During the operation of the buoyancy engine, the center of the buoyancy is shifted along the $O x_{0}$ axis by Eq. (4).

$$
\begin{aligned}
\tau_{w}=u_{b} \pi R_{c}^{2} \rho g \\
x_{b}=\frac{\pi R_{c}^{2} u_{b}\left(X_{p}+\frac{u_{b}}{2}\right)}{\pi R_{c}^{2} u_{b}+V_{n b}}
\end{aligned}
$$

where, $X_{p}$ is the position of the piston in the neutral position along the $O x_{0}$ axis in the body-fixed frame; $V_{n b}$ is the volume of the vehicle in the neutral condition of the buoyancy engine.

The movable mass in Fig. 3 can move along the $0 x_{0}$ axis; therefore, the center of gravity in this axis $x_{g}$ can be defined as:

$$
x_{g}=\frac{m_{\text {stat }} x_{s t a t}+m_{m} u_{m}}{m}
$$


where, $m_{\text {stat }}=m-m_{m}$ is the static mass; $m_{m}$ is the weight of the movable mass; $u_{m}$ is the position of the moving mass; $x_{\text {stat }}$ is the position of the static mass and it is assumed to be very small because the origin of body-fixed frame is located near to the center of gravity. Furthermore, $x_{g}$ can be approximated as:.

$$
x_{g} \approx \frac{m_{m}}{m} u_{m}
$$

The moment produced from the mass-shifter can be computed by the product of the net buoyancy force and the location of the center of gravity as found in Eq. (7). In addition, the moment of inertia is also changed because of the change in mass distribution following Eq. (8). $I_{n y}$ is the moment of inertia in the neutral condition of moving mass.

$$
\begin{aligned}
& \tau_{q}=W x_{g}=W \frac{m_{m}}{m} u_{m} \\
& I_{y y}=I_{n y}+m_{m} u_{m}^{2} \operatorname{sgn}\left(u_{m}\right)
\end{aligned}
$$

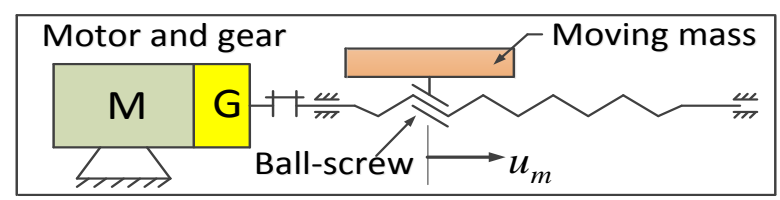

Fig. 3 Mass shifter diagram

\section{Depth Control}

\subsection{Pitch control}

The pitch angle in the proposed design of the HUG is changed by controlling the moving mass position and the pitch angle dynamics can be described as Eq. (9), which are from Eq. (2).

$$
\begin{array}{ll}
\text { System 1: } & \dot{\theta}=q \\
\text { System 2: } & m_{33} \dot{q}=f_{2}(\dot{v}, v, \eta)+\tau_{q}+\tau_{e q}
\end{array}
$$

where

$$
\begin{aligned}
& m_{33}=I_{y}-M_{\dot{q}} \\
& f_{2}=-m z_{g}(\dot{u}+q w)+m x_{g}(w-q u)+M_{\dot{w}} \dot{w}-Z_{\dot{w}} w u-Z_{\dot{q}} q u+X_{\dot{u}} u w+M_{|q| q}|q| q-\left(z_{g} W-z_{b} B\right) \sin \theta-\left(x_{g} W-x_{b} B\right) \cos \theta
\end{aligned}
$$

In this system, the buoyancy force is only controlled by on and off mode for descending and ascending. Therefore, the moment produced by moving mass is used and designed using the adaptive backstepping technique for the vertical dynamics. Two differential equations in Eq. (9) are considered as two subsystems for the backstepping control. For one equation, the virtual control law is set for $q_{d}$ so that $\theta \rightarrow \theta_{d}$ as $t \rightarrow \infty$, and then the control moment $\tau_{q}$ is designed for controlling $q \rightarrow q_{d}$ as $t \rightarrow \infty$. The errors of system 1 and 2 are defined in:

$$
\left\{\begin{array}{l}
e_{1}=\theta-\theta_{d} \\
e_{2}=q-q_{d}
\end{array}\right.
$$

Error dynamics of the pitch dynamics are defined as below:

$$
\dot{e}_{1}=\dot{\theta}-\dot{\theta}_{d}=q-\dot{\theta}_{d}=e_{2}-\dot{\theta}_{d}+q_{d}
$$


The error dynamics in Eq. (13) can be stabilized by designing $q_{d}=\dot{\theta}_{d}-k_{1} e_{1}$ with the positive control gain, $k_{1}$. Then, the error dynamics of system 1 can be obtained as:

$$
\dot{e}_{1}=-k_{1} e_{1}+e_{2}
$$

Derivative of the virtual control is:

$$
\dot{q}_{d}=\ddot{\theta}_{d}+k_{1}^{2} e_{1}-k_{1} e_{2}
$$

Error dynamics of the subsystem 2 is obtained as:

$$
m_{33} \dot{e}_{2}=m_{33} \dot{q}-m_{33} \dot{q}_{d}=f_{2}-m_{33} \dot{q}_{d}+\tau_{q}+\tau_{e q}
$$

Using the sliding mode control technique for finding the control law for pitch control, one can choose the Lyapunov function for this system as below:

$$
V_{2}=\frac{1}{2} e_{1}^{2}+\frac{1}{2} m_{33} e_{2}^{2}+\frac{1}{2} \tilde{a}_{2} P_{2}^{-1} \tilde{a}_{2}
$$

where $Y_{2} a_{2}=-f_{2}+m_{33} \dot{q}_{d} ; P_{2}=I_{9 \times 9}$ is the identity matrix; $\tilde{a}_{2}=\hat{a}_{2}-a_{2} ; Y_{2}=\left[\dot{u} \dot{w} q u w u q w|q| q \sin \theta \cos \theta \dot{q}_{d}\right]$ is the regressive vector for updating the parameter vector $a_{2}$ defined as:

$$
a_{2}=\left[-m z_{g}\left(m x_{g}-M_{\dot{w}}\right)\left(-m x_{g}+Z_{\dot{q}}\right)\left(X_{\dot{u}}-Z_{\dot{w}}\right)-m z_{g} M_{|q| q}-\left(z_{g} W-z_{b} B\right)-\left(x_{g} W-x_{b} B\right)-m_{33}\right]^{T}
$$

The derivative of the above Lyapunov function is expanded in Eq. (19). To stabilize the dynamics in this equation, the control input is designed as Eq. (20) with the saturation function in the switching control.

$$
\begin{aligned}
& \dot{V}_{2}=-k_{1} e_{1}^{2}+e_{1} e_{2}+e_{2}\left(-Y_{2} a_{2}+\tau_{q}+\tau_{e q}\right)+\dot{\hat{a}}^{T} P_{2}^{-1} \tilde{a}_{2} \\
& \tau_{q}=Y_{2} \hat{a}_{2}-k_{2} e_{2}-e_{1}-\hat{\tau}_{e q}-k_{2 \Delta} \operatorname{sat}\left(\frac{e_{2}}{\phi_{2}}\right)
\end{aligned}
$$

Substituting the control law (20) into the first derivative of the Lyapunov function (19) will result to:

$$
\dot{V}_{2}=-k_{1} e_{1}^{2}-k_{2} e_{2}^{2}-k_{2 \Delta} e_{2} \operatorname{sat}\left(\frac{e_{2}}{\phi_{2}}\right)+e_{2}\left(\tau_{e q}-\hat{\tau}_{e q}\right)+e_{2} Y_{2} \tilde{a}_{2}+\dot{\hat{a}}_{2} P_{2}^{-1} \tilde{a}_{2}
$$

By choosing the adaption law as $\dot{\hat{a}}=-P_{2} Y_{2}^{T} e_{2}$, the derivative of $V_{2}$ can be derived as:

$$
\dot{V}_{2}=-k_{1} e_{1}^{2}-k_{2} e_{2}^{2}-k_{2 \Delta} e_{2} \operatorname{sat}\left(\frac{e_{2}}{\phi_{2}}\right)+e_{2}\left(\tau_{e q}-\hat{\tau}_{e q}\right)
$$

It is observed that if $k_{2 \Delta}>F_{e q}$ and $\left|\tau_{e q}-\hat{\tau}_{e q}\right| \leq F_{e q}$, then $\dot{V}_{2} \leq-k_{1} e_{1}^{2}-k_{2} e_{2}^{2} \leq 0$. Here, $F_{e q}$ is defined relating with the environmental condition. Following the direct Lyapunov method for $V_{2}$, the control law (20) is proved to be globally asymptotically stable (GAS).

\subsection{Speed control}

The vehicle speed will be controlled by thruster attached at the tail of HUG. The speed dynamics will be investigated to carry out the speed controller. The system 3 is defined as Eq. (23) which is the 4th equation in Eq. (2). 
System 3: $m_{11} \dot{u}=f_{3}(\dot{v}, v, \eta)+\tau_{w} \sin \theta+\tau_{u}+\tau_{e u}$

where

$$
\begin{aligned}
& m_{11}=m-X_{\dot{u}} \\
& f_{3}=-m z_{g} \dot{q}+\left(m x_{g}+Z_{\dot{q}}\right) q^{2}-\left(m-Z_{\dot{w}}\right) w q+X_{|u| u}|u| u-(W-B) \sin \theta
\end{aligned}
$$

The speed error is defined as:

$$
e_{3}=u-u_{d}
$$

And the speed error dynamics can be formulated as:

$$
m_{11} \dot{e}_{3}=m_{11} \dot{u}-\dot{u}_{d}=f_{3}+\tau_{w} \sin \theta+\tau_{u}-m_{11} \dot{u}_{d}
$$

The Lyapunov candidate can be designed as:

$$
V_{3}=\frac{1}{2} m_{11} e_{3}^{2}+\frac{1}{2} \tilde{a}_{3}^{T} P_{3}^{-1} \tilde{a}_{3}
$$

where

$$
\begin{aligned}
& a_{3}=\left[-m z_{g}\left(m x_{g}+Z_{\dot{q}}\right)\left(-m+Z_{\dot{w}}\right) X_{|u| u}-(B-W) \tau_{w}-m_{11}\right]^{T} \\
& \tilde{a}_{3}=\hat{a}_{3}-a_{3}
\end{aligned}
$$

Furthermore, its derivative is formulated as Eq. (31) using the dynamics in Eq. (27).

$$
\dot{V}_{3}=e_{3}\left(-Y_{3} a_{3}+\tau_{u}+\tau_{e u}\right)+\tilde{a}_{3} P_{3}^{-1} \tilde{a}_{3}
$$

where $Y_{3}=\left[\dot{q} q^{2} w q|u| u \sin \theta \dot{u}_{d}\right]$. The speed control law can be designed as:

$$
\tau_{u}=Y_{3} \hat{a}_{3}-k_{3} e_{3}-\hat{\tau}_{e u}-k_{3 \Delta} \operatorname{sat}\left(\frac{e_{3}}{\phi_{3}}\right)
$$

Applying this control law into (31) yields:

$$
\dot{V}_{3}=-k_{3} e_{3}^{2}-k_{3 \Delta} \operatorname{sat}\left(\frac{e_{3}}{\phi_{3}}\right) e_{3}+e_{3}\left(\tau_{e u}-\hat{\tau}_{e u}\right)+e_{3} Y_{3} \tilde{a}_{3}+\dot{a}^{T} P_{3}^{-1} \tilde{a}_{3}
$$

The last two term in Eq. (25) can be eliminated by choosing $\dot{\hat{a}}_{3}=-P_{3} Y_{3}^{T} e_{3}$, then $\dot{V}_{3}$ can be reformulated as:

$$
\dot{V}_{3}=-k_{3} e_{3}^{2}-k_{3 \Delta} \operatorname{sat}\left(\frac{e_{3}}{\phi_{3}}\right) e_{3}+e_{3}\left(\tau_{e u}-\hat{\tau}_{e u}\right)
$$

If the control gain $k_{3 \Delta}$ is chosen as:

$$
k_{3 \Delta}>\left|\tau_{e u}-\hat{\tau}_{e u}\right|
$$

Then, the derivative of the Lyapunov function $V_{3}$ is negative definite as: 


$$
\begin{aligned}
\dot{V}_{3} & \leq-k_{3} e_{3}^{2}-k_{3 \Delta}\left|e_{3}\right|+\left|e_{3}\right|\left|\tau_{e u}-\hat{\tau}_{e u}\right| \\
& \leq-k_{3} e_{3}^{2} \quad \text { (N.D) }
\end{aligned}
$$

This control law is proved to be GAS using Lyapunov function $V_{3}$ as shown in Eq. (28).

\subsection{LOS depth-keeping guidance}

For navigation of the HUG, a line of sight (L.O.S) method based on the calculated heading angle was used [18]. The line-of-sight (LOS) guidance in Fig. 4 is applied for path following control for the vertical plane. It is assumed that the position information of the HUG is available from the inertial navigation system. Then, a two-dimensional LOS guidance is constructed to apply the desired pitch angle $\theta_{d}$ to the controller. The way-point in the LOS guidance is given by the operator and it contains 2 components, $x_{k}$ and $z_{k}$. The path variables by the previous waypoint and the current waypoint are defined by $x_{k}, z_{k}, x_{k-1}$ and $z_{k-1}$. By solving Eqs. (37)-(38) for the virtual LOS point $\left(x_{l o s}, z_{l o s}\right)$, the desired pitch angle can be computed by Eq. (39). $L_{p p}$ is the length of the vehicle; $n$ is the positive gain that combines with $L_{p p}$ to define the LOS radius.

$$
\begin{aligned}
& \left(z_{\text {los }}-z\right)^{2}+\left(x_{l o s}-x\right)^{2}=\left(n L_{p p}\right)^{2} \\
& \frac{z_{l o s}-z_{k-1}}{x_{l o s}-x_{k-1}}=\frac{z_{k}-z_{k-1}}{x_{k}-x_{k-1}} \\
& \theta_{d}=\arctan \left(\frac{z_{l o s}-z}{x_{l o s}-x}\right)
\end{aligned}
$$

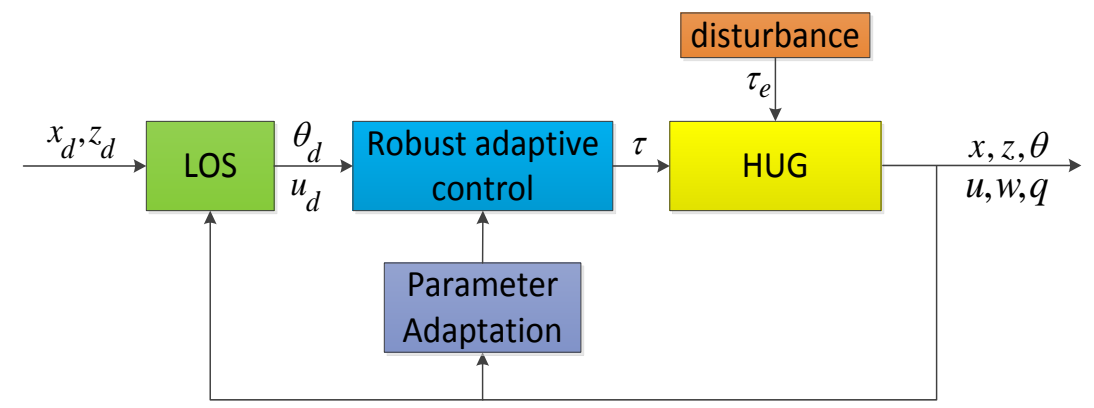

Fig. 4 Algorithm of depth control

For the energy efficient operation, HUG will glide down by only using the buoyancy engine and mass shifter. When it reaches the desired depth, the depth control is applied to keep it at the desired depth. The adaptation law is used to estimate the nonlinear unknown term in the pitch and surge dynamics. The estimation algorithm for the unknown parameters is used in the control action while stabilizing the dynamics. Furthermore, the sliding mode control is used to deal with the environmental disturbances from currents and waves. In the following section, the simulation of the diving, depth-keeping and redurfacing are presented.

\section{Simulation and Discussion}

In order to validate the good performance of the proposed depth control, the simulation of heaving motion control of the HUG is presented using the MATLAB with the vehicle parameters which is presented in Table 1. The descending and ascending motion is actuated by only buoyancy engine and mass shifter. When the HUG goes down close to the desired depth, the buoyancy engine is controlled to stay in the neutral buoyant condition or zero net buoyancy. The thruster and mass shifter are used to drive the vehicle along the desired depth with the designed speed. In this simulation, the desired depth is $200 \mathrm{~m}$ 
$\left(z_{1}=z_{2}=200 \mathrm{~m}\right)$, the length of trajectory distance is $300 \mathrm{~m}$, the desired speed is 2 knots $\left(u_{d}=1 \mathrm{~m} / \mathrm{s}\right)$. Also, environmental

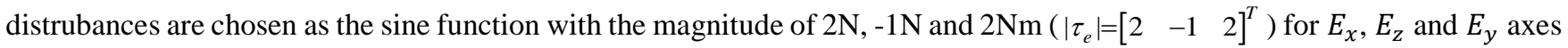
respectively.

Table 1 Vehicle parameters

\begin{tabular}{|c|c|c|c|c|c|}
\hline Parameters & Value & Parameters & Value & Parameters & Value \\
\hline $\mathrm{m}$ & $70 \mathrm{~kg}$ & $X_{\dot{u}}$ & $-9.3 \times 10^{-1} \mathrm{~kg}$ & $Z_{|w| w}$ & $-1.31 \times 10^{2} \mathrm{~kg} / \mathrm{m}$ \\
\hline$I_{y y}$ & $1.29 \mathrm{kgm}{ }^{2}$ & $Z_{\dot{w}}$ & $-3.55 \times 10^{1} \mathrm{~kg}$ & $M_{\dot{q}}$ & $-4.88 \mathrm{kgm}^{2} / \mathrm{rad}$ \\
\hline$m_{m}$ & $1.5 \mathrm{~kg}$ & $Z_{\dot{q}}$ & $-1.93 \mathrm{kgm} / \mathrm{rad}$ & $M_{\dot{w}}$ & $-1.93 \mathrm{kgm}$ \\
\hline$W-B$ & $\pm 0.7 \mathrm{~kg}$ & $X_{|u| u}$ & $-3.9 \mathrm{~kg} / \mathrm{m}$ & $M_{|q| q}$ & $-1.88 \times 10^{2} \mathrm{kgm}^{2} / \mathrm{rad}^{2}$ \\
\hline
\end{tabular}

The HUG dives with $-30^{\circ}$ pitch angle to the depth of $200 \mathrm{~m}$, then it will use the thruster to keep at the constant depth, and after moving $300 \mathrm{~m}$ at the desired depth it will ascends with a positive pitch angle of $30^{\circ}\left(\theta_{d}= \pm 30^{\circ}\right)$. Fig. 5 is indicated that HUG glides down with the small oscillation due to the environment effects. This phenomenon can be observed in the pitch tracking control in Fig. 6. At the desired depth, the output of LOS guidance is seen as the wave form because of the sine function of the disturbances. It shows that the control law drives the HUG to track the path of LOS guidance very well in the presence of disturbances.

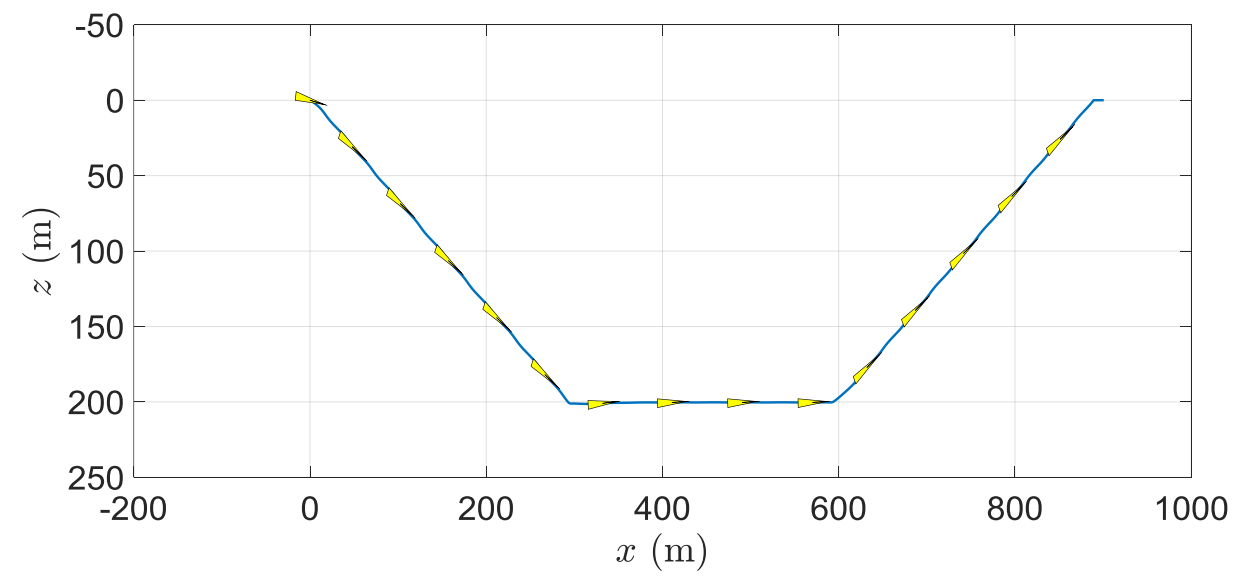

Fig. 5 Trajectory of HUG with environmental disturbances

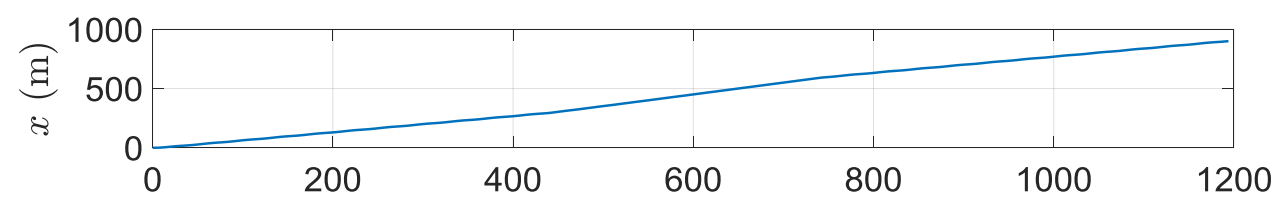

(a) longitudinal coordinate

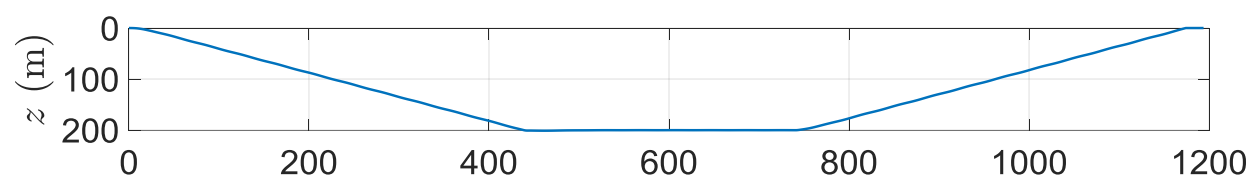

(b) depth

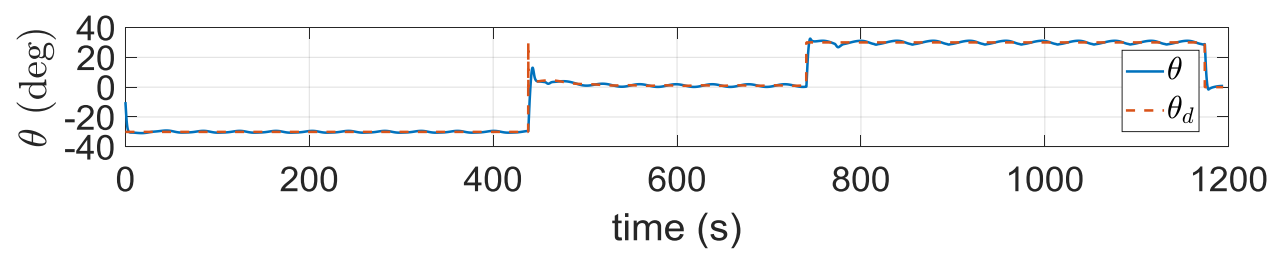

(c) heading performance in depth control

Fig. 6 The position and orientation of HUG 
Fig. 7 is described velocities of the vehicle in the body-fixed coordinates. The speed control results are presented for the surge velocity $u$ with the desired speed is $1 \mathrm{~m} / \mathrm{s}$. It is noted that the speed control is only used in the cruise period. The robust adaptive speed control shows good tracking performance along the desired trajectory under the bounded disturbances. Additionally, the control force and torque for the depth control are simulated in Fig. 8. The control force of the buoyancy engine is limited in the range from $-2 \mathrm{~N}$ to $2 \mathrm{~N}$. The range of the pitching torque generated by mass shifter is in the range from $-20 \mathrm{Nm}$ to $20 \mathrm{Nm}$.

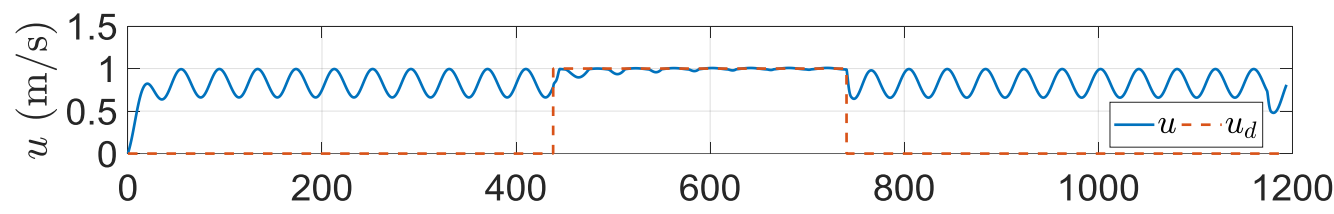

(a) speed control performance

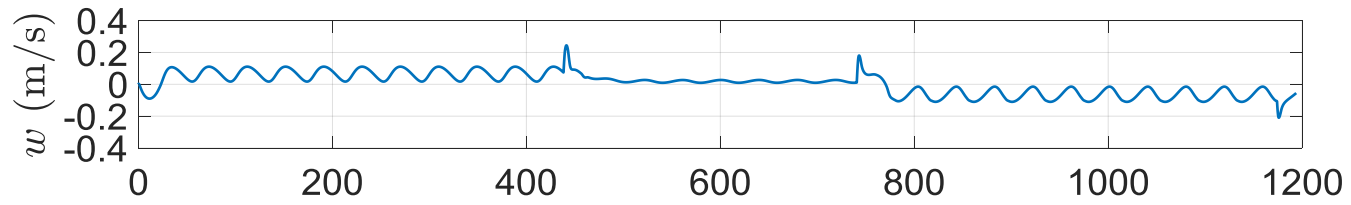

(b) sway velocity

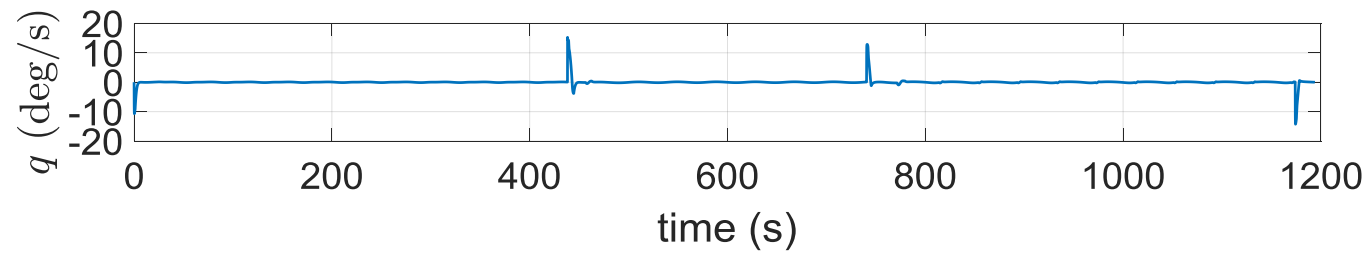

(c) pitch velocity

Fig. 7 Velocities of the HUG in the body-fixed coordinates

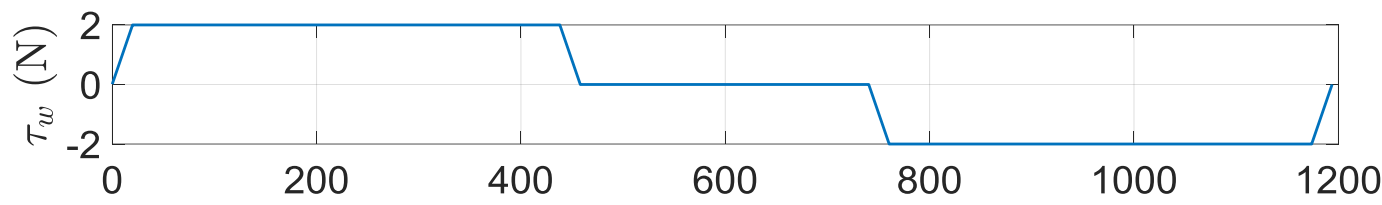

(a) net buoynacy force

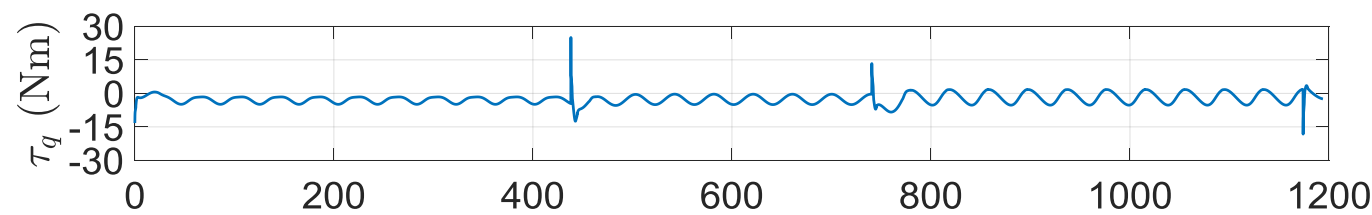

(b) moment induced by moving mass

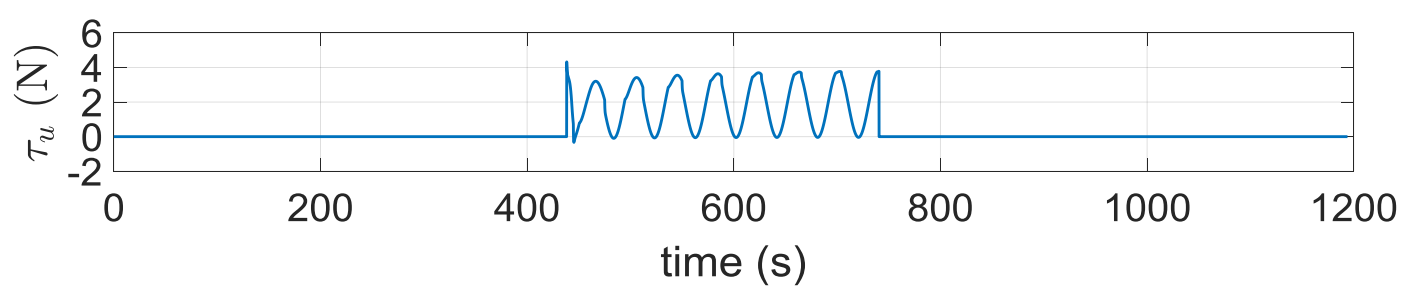

(c) thruster force during the depth control

Fig. 8 Force and moment from the robust adaptive controller

As stated above, the stability proof is revealed that the errors are bounded in Fig. 9. $e_{1}$ and $e_{2}$ are from pitching control system, and they show good convergence performance, even though the desired angle changes due to the LOS guidance and external disturbance effects. Especially, during the transition period from gliding to cruising or vice versa, $e_{1}$ and $e_{2}$ can quickly converge near to zero in Fig. 9. For speed control, the convergence only appears in the cruising mode while keeping the 


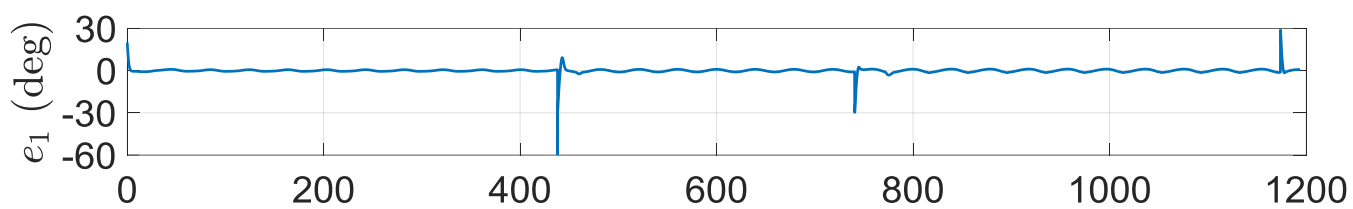

(a) pitch angle error ( $e_{1}$ is pitch angle error)

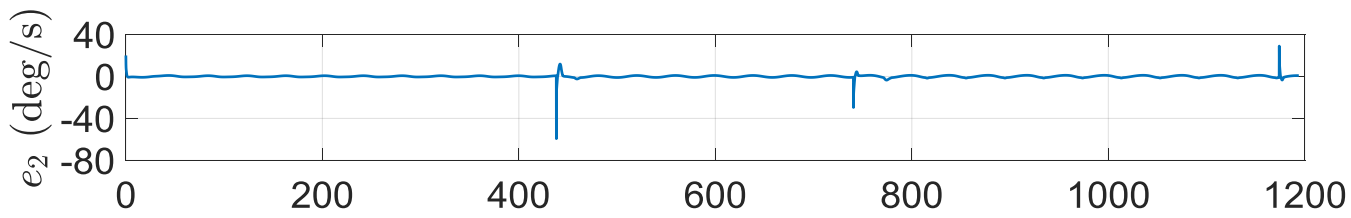

(b) virtual control error ( $e_{2}$ is the virtual control error)

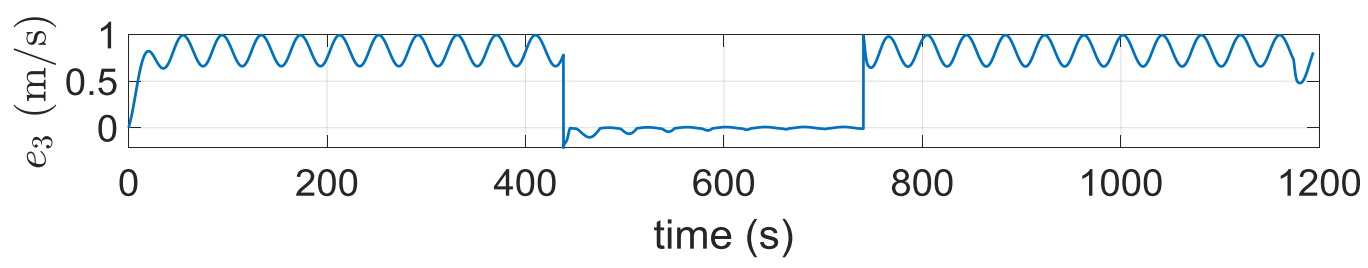

(c) speed control error in the desired depth ( $e_{3}$ is the speed control error)

Fig. 9 The tracking errors from 3 sub-controller in crusing period

HUG to the desired depth of $200 \mathrm{~m}$. In the desired depth, the speed of the vehicle is controlled at a constant value of $1 \mathrm{~m} / \mathrm{s}$ or 2 knots. At the same time, the error converges quickly close to zero in Fig. 9.

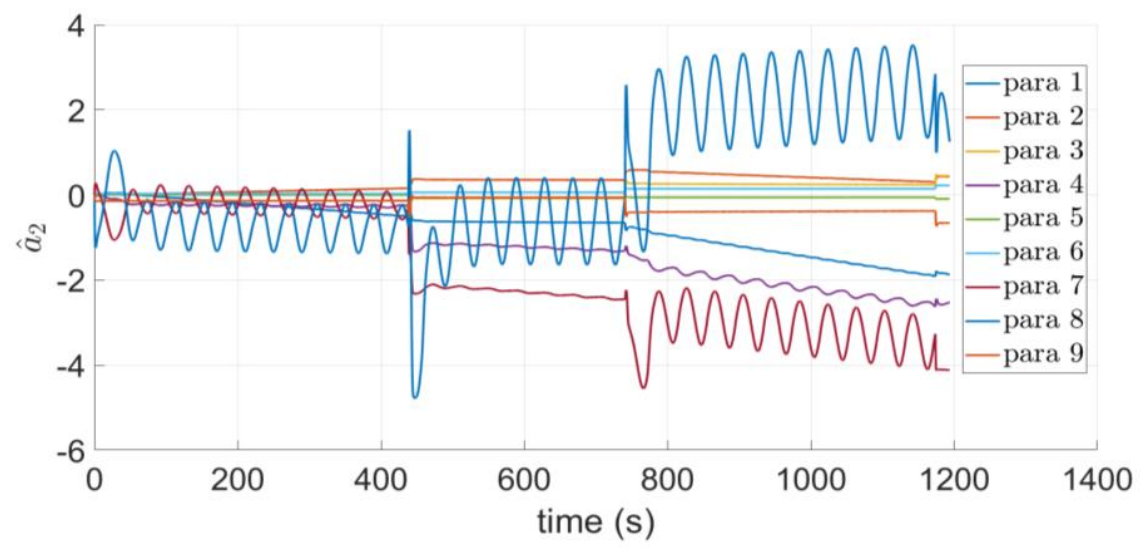

Fig. 10 The parameter adaptation of vector $\hat{a}_{2}$

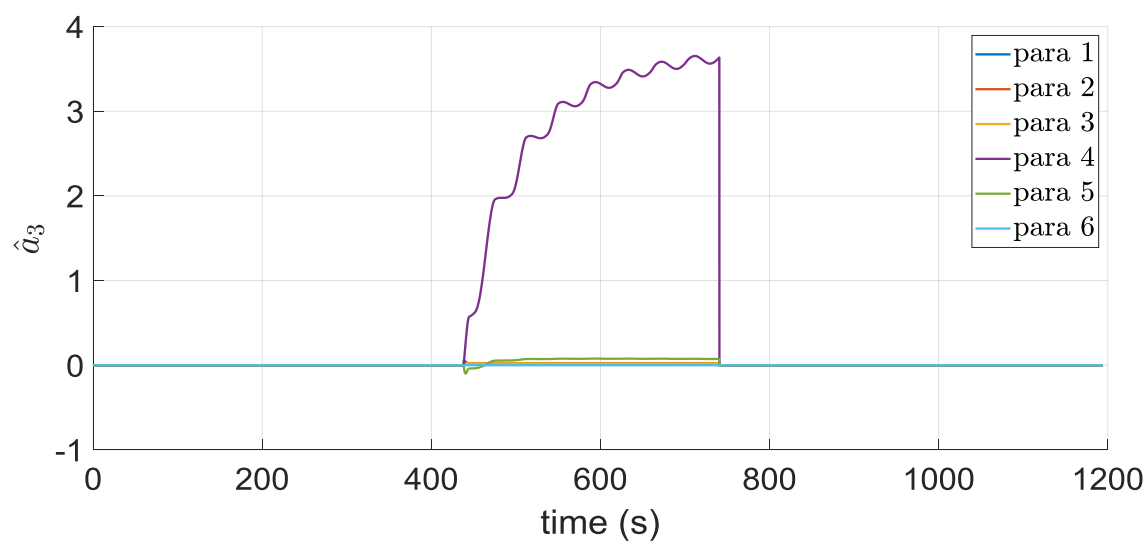

Fig. 11 The parameter adaptation of vector $\hat{a}_{3}$

Two main adaptation processes are indicated in Fig. 10 for vector $a_{2}$ and $a_{3}$ respectively. There are 9 parameters in vector $a_{2}$ (18) and 6 parameters in vector $a_{3}$ (29). Parameters 7 and 8 of vector $a_{2}$ in Fig. 10 are sensitive to the external disturbances with the wave curve updating process. These parameters are defiend as $-\left(z_{g} W-z_{b} B\right)$ and $\left(x_{g} W\right.$ - $\left.x_{b} B\right)$ respectively in 
(18). These terms are from the restoring moment due to the change of the gravity center and buoyancy center. In addition, in vector $a_{3}$, there is one parameter that proportionally adapts to the change of disturbances, which is parameter 4 defined as $X_{|u| u}$ in the damping force in the system 3. This phenomenon is shown in Fig. 11, parameter 4 shows the waving curve in the speed control.

\section{Conclusions}

In this study, the depth control for nonlinear vertical dynamics of the hybrid underwater glider was proposed and its performance was validated through simulations. The robust adaptive control law has presented the stable performance against the parameter change and external disturbance. It is guaranteed that the error is close to zero as the time go to infinite. According to the direct Lyapunov method, the stability of this control law has been established and proved. The depth control algorithm uses the LOS guidance law for keeping the HUG to the desired depth. The computer simulation using MATLAB was performed to verify the good and stable performance of depth keeping control using the proposed control algorithm. In the future research, this algorithm will be implemented in the developed HUG at the laboratory and, the water tank and sea trials of the HUG employing the proposed depth control will be carried out.

\section{Acknowledgement}

This study is part of a project supported by the Civil-Military Dual-Use Technology "Data Collection System with Underwater Glider" (Grant No. 19-SN-MU-01) and a government project entitled "Development of hybrid underwater drone for accurate inspection of the underwater pipeline and cable".

\section{Conflicts of Interest}

The authors declare no conflict of interest

\section{References}

[1] M. G. Joo and Z. Qu, “An autonomous underwater vehicle as an underwater glider and its depth control,” International Journal of Control, Automation and Systems, vol. 13, no. 5, pp. 1212-1220, July 2015.

[2] I. Abraham and J. Yi, "Model predictive control of buoyancy propelled autonomous underwater glider," Proc. of the American Control Conference, July 2015, pp. 1181-1186.

[3] S. K. Jeong, H. S. Choi, J. H. Bae, S. S. You, H. S. Kang, S. J. Lee, et al., "Design and control of high speed unmanned underwater glider," International Journal of Precision Engineering and Manufacturing-Green Technology, vol. 3, no. 3, pp. 273-279, July 2016.

[4] B. Claus and R. Bachmayer, "Energy optimal depth control for long range underwater vehicles with applications to a hybrid underwater glider,” Autonomous Robots, vol. 40, no. 7, pp. 1307-1320, February 2016.

[5] M. Mat-Noh, M. R. Arshad, and R. Mohd-Mokhtar, "Nonlinear control of autonomous underwater glider based on super-twisting sliding mode control (STSMC)," 7th IEEE International Conference on System Engineering and Technology, October 2017, pp. 71-76.

[6] R. D. S. Tchilian, E. Rafikova, S. A. Gafurov, and M. Rafikov, "Optimal control of an underwater glider vehicle," Procedia Engineering, vol. 176, pp. 732-740, 2017.

[7] Y. Liu, J. Ma, N. Ma, and G. Zhang, "Path planning for underwater glider under control constraint," Advances in Mechanical Engineering, vol. 9, no. 8, pp. 1-9, August 2017.

[8] Z. Huang, H. Zheng, S. Wang, Y. Liu, J. Ma, and Y. Liu, "A self-searching optimal ADRC for the pitch angle control of an underwater thermal glider in the vertical plane motion," Ocean Engineering, vol. 159, pp. 98-111, July 2018.

[9] Z. Q. Su, M. Zhou, F. F. Han, Y. W. Zhu, D. L. Song, and T. T. Guo, “Attitude control of underwater glider combined reinforcement learning with active disturbance rejection control,” Journal of Marine Science and Technology, vol. 24, no. 3, pp. 686-704, 2019. 
[10] H. Sang, Y. Zhou, X. Sun, and S. Yang, "Heading tracking control with an adaptive hybrid control for under actuated underwater glider,” ISA Transactions, vol. 80, no. 10, pp. 554-563, July 2018.

[11] D. Song, T. Guo, W. Sun, Q. Jiang, and H. Yang, "Using an active disturbance rejection decoupling control algorithm to improve operational performance for underwater glider applications," Journal of Coastal Research, vol. 34, no. 3, p. 724, May 2018.

[12] M. G. Joo, “A controller comprising tail wing control of a hybrid autonomous underwater vehicle for use as an underwater glider," International Journal of Naval Architecture and Ocean Engineering, vol. 11, no. 2, pp. 865-874, July 2019.

[13] S. K. Jeong, H. S. Choi, J. Il Kang, J. Y. Oh, S. K. Kim, and T. Q. M. Nhat, "Design and control of navigation system for hybrid underwater glider,” Journal of Intelligent and Fuzzy Systems, vol. 36, no. 2, pp. 1057-1072, March 2019.

[14] N. D. Nguyen, H. S. Choi, and S. W. Lee, "Robust adaptive heading control for a ray-type hybrid underwater glider with propellers," Journal of Marine Science and Engineering, vol. 7, no. 10, October 2019.

[15] T. T. J. Prestero, "Verification of a six-degree of freedom simulation model," PhD Thesis, Dept. Mechanical Engineering, University of California at Davis, 2001.

[16] D. H. Ji, H. S. Cho, S. K. Jeong, J. Y. Oh, S. K. Kim, and S. S. You, "A study on heading and attitude estimation of underwater track vehicle,” Advances in Technology Innovation, vol. 4, no. 2, pp. 84-93, April 2019.

[17] Handbook of Marine Craft Hydrodynamics and Motion Control, John Wiley \& Sons, 2011.

[18] D. Jung, S. Hong, J. Lee, H. Cho, H. Choi, and M. Vu, "A study on unmanned surface vehicle combined with remotely operated vehicle system," Proceedings of Engineering and Technology Innovation, 2018, vol. 9, no. 7, pp. 17-24, July 2018.

Copyright $\odot$ by the authors. Licensee TAETI, Taiwan. This article is an open access article distributed under the terms and conditions of the Creative Commons Attribution (CC BY-NC) license (https://creativecommons.org/licenses/by-nc/4.0/). 